\title{
Manipulation of viscous all-aqueous jets by electrical charging
}

\author{
Yang Song ${ }^{\mathrm{a}, \mathrm{b}}$, Zhou Liu ${ }^{\mathrm{a}, \mathrm{b}}$, Tiantian Kong ${ }^{\mathrm{a}, \mathrm{b}}$ and Ho Cheung Shum ${ }^{\mathrm{a}, \mathrm{b} *}$ \\ Received (in $X X X, X X X)$ Xth $X X X X X X X X X 20 X X$, Accepted Xth $X X X X X X X X X 20 X X$ \\ DOI: 10.1039/b000000x
}

5 We demonstrate the manipulation of viscous all-aqueous jets by electrical charging. At sufficiently high voltages, the folding instability of an uncharged viscous jet is suppressed, and the jet diameter can be adjusted by varying the applied voltage or the fluid flow rates. This inspires new ways to 10 fabricate biocompatible fibers.

Hydrogel fibers have attracted growing interests in the fabrication of biomaterials and tissue engineering, for instance, as matrix materials in cell culture ${ }^{[1]}$ and as tissue scaffolds ${ }^{[2]}$. Conventional techniques for fabricating hydrogel fibers include 15 electrospinning $^{[3]}$, extrusion ${ }^{[4]}$, mold casting ${ }^{[5]}$ and ink-jet printing $^{[6]}$. Recently, multiphase microfluidic devices have been introduced to generate materials with more complex structures and inner architectures ${ }^{[7]}$. However, these multiphase approaches often include the use of organic solvents that are harmful to 20 biological cells and can lead to denaturation of bioactive molecules. Therefore, there is a need for multiphase systems that are completely free of organic solvents. One promising candidate is aqueous two-phase systems (ATPS) ${ }^{[8]}$, which consists of two or more immiscible aqueous phases with mutually incompatible ${ }_{25}$ dissolved additives. Due to the aqueous nature of these phases, ATPS typically has a low interfacial tension of less than 1 $\mathrm{mN} / \mathrm{m}^{[9]}$. Due to the reduction in the driving force for capillary instability, the low tension facilitates formation of water-in-water $(\mathrm{w} / \mathrm{w})$ jets $^{[10]}$, which are excellent templates for hydrogel fibers ${ }^{[11]}$ ${ }_{30}$ Common ATPSs consist of concentrated solutions of macromolecular solutes above their critical concentrations for codissolution ${ }^{[8,9]}$. These concentrated solutions usually have high viscosities, making them difficult to manipulate precisely by hydrodynamic force alone in microchannels ${ }^{[12]}$. To allow the use 35 of these w/w jets as templates for biocompatible fibers and complex soft structures, a novel approach to manipulate these viscous all-aqueous jets is desired.

In this work, as an alternative to hydrodynamic force, we apply electrostatic force to control the deformation of the viscous all40 aqueous jets. To form a w/w jet, two immiscible aqueous phases with different electrical conductivities are injected into a coflowing microfluidic device (figure 1a). The inner jet phase is an aqueous mixture of $10 \mathrm{wt} \%$ dextran $\left(M_{w}=5,00,000\right)$ and $10 \mathrm{wt} \%$ dextran sodium sulfate $\left(M_{w}=5,00,000\right)$ solutions; the outer 45 continuous phase is a $13 \mathrm{wt} \%$ PEG $\left(M_{w}=8000\right)$ solution. The electrical conductivities of the jet phase and the continuous phases are $8300 \mu \mathrm{S} / \mathrm{cm}$ and $55 \mu \mathrm{S} / \mathrm{cm}$ respectively. At typical volumetric flow rates, the jet forms a periodic folding structure (see figure $2 \mathrm{a}$ and the state diagram of figure $\mathrm{S} 1$ in the supporting 50 information). This folding structure is induced by the compressive stress along the flow direction when the jet decelerates $^{13}$; as a result, the highly viscous jet $\left(\eta_{\text {in }} / \eta_{\text {out }}>100\right)$ tends to minimize dissipation by folding, rather than expansion ${ }^{13}$.
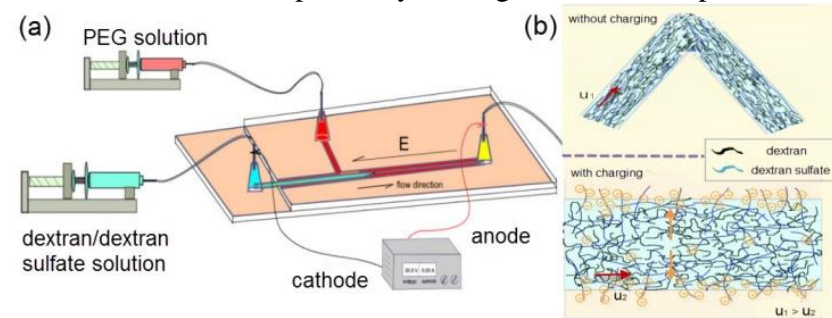

${ }_{55}$ Fig.1 (a) Experimental setup of the microfluidic device incorporated with a DC electric field along its microchannel. (b) Schematics of jet expansion upon electric charging: electrostatic force induces dextran sulfate to migrate to the w/w interface.

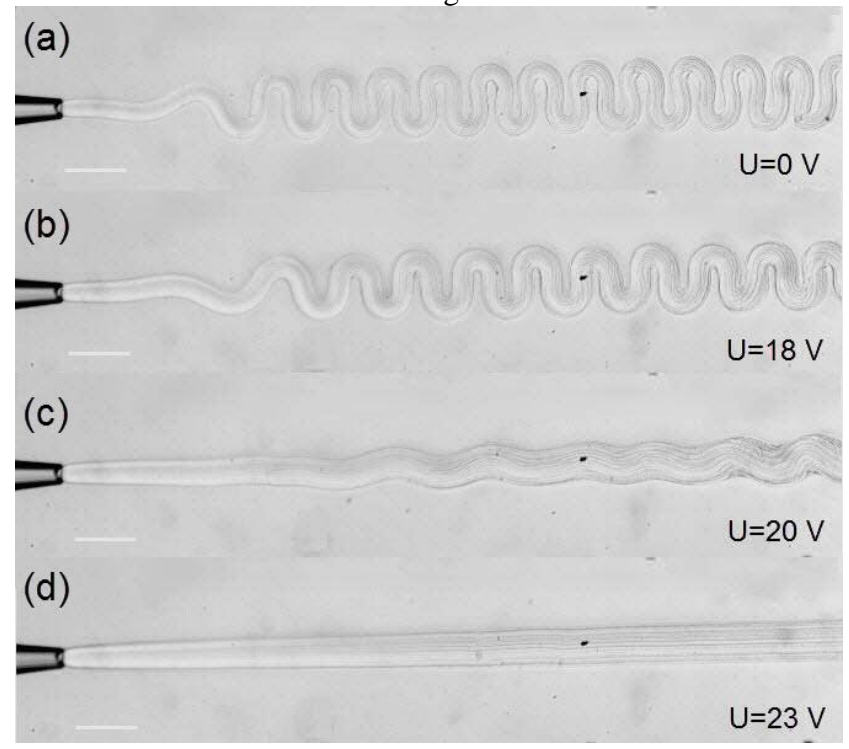

${ }_{60}$ Fig.2 Optical microscope images of a viscous jet of a mixture of $10 \%$ dextran and $10 \%$ dextran sulfate solutions in a continuous phase of a $13 \%$ PEG solution, captured by a high speed camera. (a) Viscous folding of the w/w jet at $0 \mathrm{~V}$; (b-d) the jet unfolds as the applied voltage increases. At a critical voltage, $U_{c}{ }^{*}$, the folded 65 jet is straightened. The flow rates are as follows: $Q_{i n}=200 \mu \mathrm{L} / \mathrm{h}$ and $Q_{\text {out }}=5000 \mu \mathrm{L} / \mathrm{h}$; dimensions of the channel: $1 \times 1 \mathrm{~mm}$; Scale bars represent $200 \mu \mathrm{m}$.

Consequently, the viscous jet maintains a constant diameter 70 despite changes in the flow rates; this behavior is similar to that of a solid wire immersed in a fluid. For example, when the flow rate of the inner jet phase, $Q_{i n}$, is fixed at $200 \mu \mathrm{L} / \mathrm{h}$, and the flow rate of the continuous phase, $Q_{\text {out }}$, increases from $2000 \mu \mathrm{l} / \mathrm{h}$ to $15000 \mu \mathrm{l} / \mathrm{h}$, the resultant jet changes in diameter by less than $5 \%$. 75 Under similar experimental conditions, a less viscous jet of the 10 
wt $\%$ dextran solution shows a reduction in diameter by $73 \%$ (see figure S2 in the supporting information). This highlights the difficulty in varying the size of viscous jets through changing fluid flow rates alone.

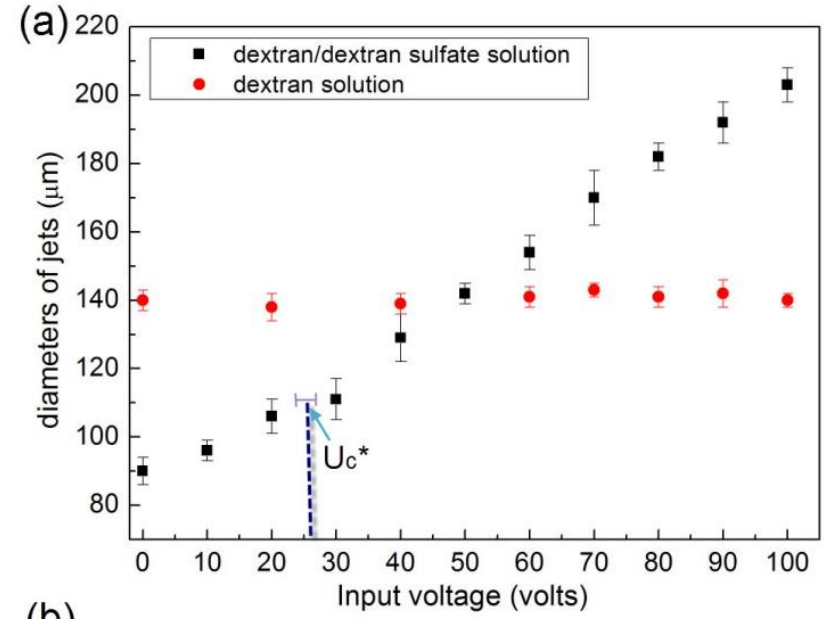

(b)

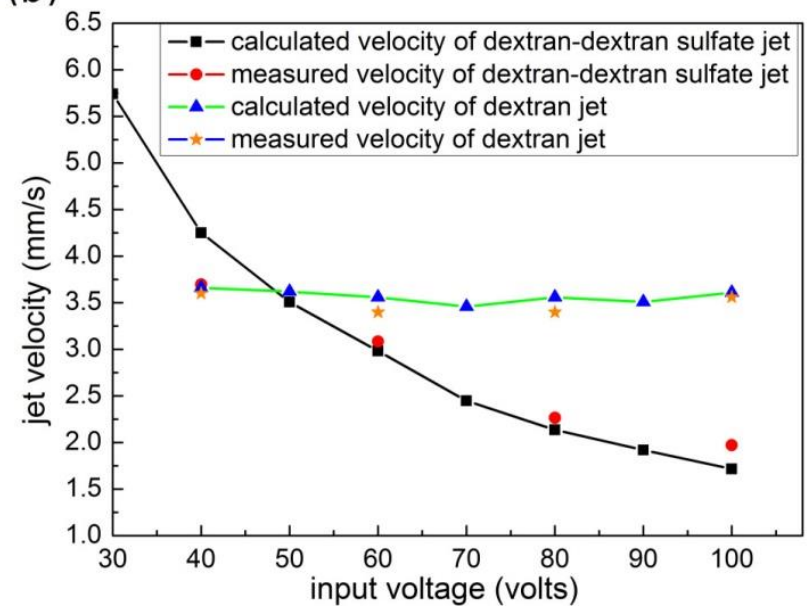

Fig.3 (a) A plot of the jet diameter as a function of the applied voltage. As the applied voltage increases, the jet of $10 \%$ dextran solution maintains its diameter, as shown by the red dots; while the jet of the mixture of $10 \%$ dextran and $10 \%$ dextran sulfate 10 solution expands, as drawn by the square symbols. (b) A plot of the jet of dextran and dextran sulfate solutions, which decelerates with increasing voltage. The calculated velocity $(\boldsymbol{\square})$ matches well with the measured velocity $(\mathbf{O})$. In comparison, the jet of dextran solution maintains the same velocity at different voltages,

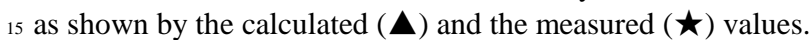

To enhance the deformability of the viscous jet, we impose an electric field parallel to the microfluidic channel (Fig.1a). Two metal needles are separately built in the device as the inlet of the 20 jet phase and the outlet of the continuous phase. The distance between the two needles is $40 \mathrm{~mm}$, and the tip of injection capillary of the jet phase is $20 \mathrm{~mm}$ away from each needle. By connecting the two needles to a regulated direct-current (DC) power supply ( $\mu$-TEK instrument PS-10010D), the two aqueous 25 phases are charged oppositely.

As the applied voltage increases, the jet of the mixture of dextran and dextran sulfate solutions widens with a responsive time scale of $10^{-1} \mathrm{~s}$; meanwhile, the frequency and amplitude of the viscous folding are also reduced (figure 2b-2c). Eventually, 30 the jet becomes unfolded and straightened above a critical voltage, $U_{c} *$ (figure $2 \mathrm{~d}$ ). This critical voltage $U_{c} *$ increases with the flow rate of the jet phase $Q_{\text {in }}$, as shown in figure $S 3$ in the supporting information. Upon further increase in voltage above $U_{c}{ }^{*}$, the jet continues to expand in diameter, as shown by the plot of the jet 35 diameter as a function of the applied voltage in figure $3 \mathrm{a}$. According to mass conservation, the jet decelerates as its diameter increases; this follows the relationship: $u_{j e t}=$ $4 Q_{i n} /\left(\pi d_{j e t}{ }^{2}\right)$. To verify the change in jet velocity $u_{j e t}$, small particles are added, as tracers, to the jet phase, and motion of the 40 particles is monitored by using a high-speed camera (Phantom V9.1). The jet velocity measured using this method of microparticle image velocimetry $(\mu \mathrm{PIV})^{[14]}$ matches well with the predicted jet velocity, as shown in figure $3 \mathrm{~b}$.

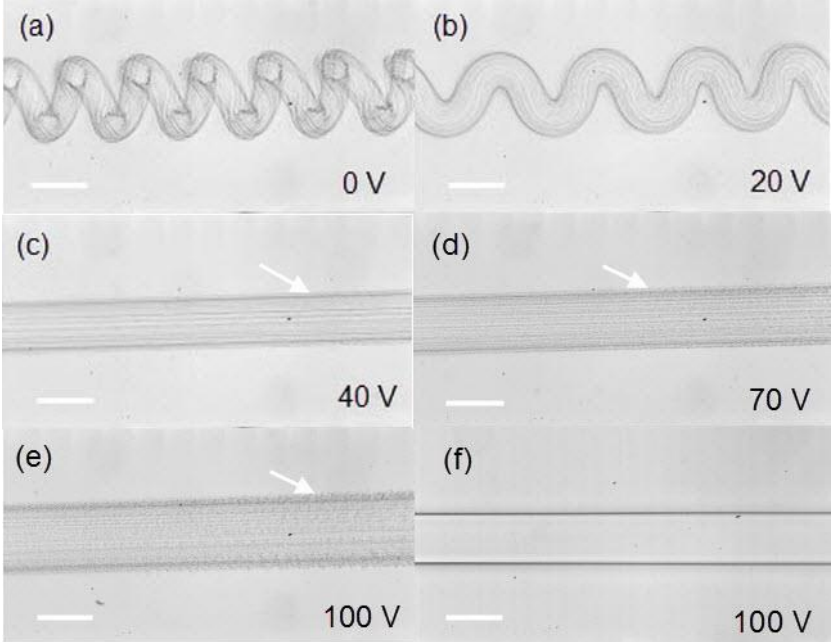

${ }_{45}$ Fig.4 Optical microscope images showing unfolding and expansion of the charged w/w jet, as captured by a high-speed camera. (a-e) The jet of the mixture of dextran and dextran sulfate solutions expands in diameter when a sufficiently high voltage is applied. A diffuse layer of dextran sulfate is observed at the 50 interface at high applied voltages (e.g. $40 \mathrm{~V}, 70 \mathrm{~V}$ and $100 \mathrm{~V}$ ), as shown by the white arrows. (f) The $10 \%$ dextran solution forms a straight jet without a diffuse layer. $Q_{i n}=250 \mu \mathrm{L} / \mathrm{h}, Q_{\text {out }}=5000 \mu \mathrm{L} / \mathrm{h}$. Scale bars represent $200 \mu \mathrm{m}$.

55 We attribute the jet expansion to migration of the charged dextran sulfate molecules inside the jet, drawn by the schematic in figure $1 \mathrm{~b}$. As a polyelectrolyte, dextran sulfate sodium dissociates in water and enhances the electrical conductivity of the jet phase. When a voltage is applied, the charged dextran ${ }_{60}$ sulfate ions in the jet phase migrate towards the interface to maximize the separation among them, thus minimize electrostatic repulsion. Since dextran sulfate cannot be dissolved in the continuous phase of the PEG solution, the migrated dextran sulfate aggregates into a concentrated diffuse layer at the 65 interface, as indicated by the arrows in figure $4 c-4 e$. This diffuse layer of the charged dextran sulfate gradually grows in thickness downstream. As the applied voltage increases, the charge density also increases. The increased electrical potential on the surface of the jet causes the jet to swell, analogous to the inflation of a 70 hollow tube with an elastic wall. The observed jet diameter represents a balance among the electrostatic repulsion, the viscous force imposed by the continuous phase and the interfacial tension. Under the same flow conditions, the viscous force remains the same; however, as the applied voltage increases, the 75 electrostatic repulsion increases, resulting in a larger jet diameter. When the interfacial tension between the jet and the continuous phases is reduced by decreasing the concentration of PEG in the continuous phase, the resultant jet with a lower interfacial tension widens more sharply upon charging than one with a higher 
interfacial tension does.

The unfolding of the charged jet can also be explained within this conceptual framework. The uncharged jets become folded because of the lower dissipation associated with folding in 5 comparison to widening in diameter. However, with the charged jet, electrostatic repulsion causes the jet to swell; consequently, at sufficiently high applied voltages and jet diameter, folding of the jet is no longer needed for mass conservation.

To confirm the role of dextran sulfate in jet swelling, we repeat 10 the experiments using a jet phase with a lower concentration of dextran sulfate, and the resultant jet swells less sensitively to the applied voltage. When pure dextran solutions are used as the jet phases, without any dextran sulfate, the jets maintain the same diameters despite electrical charging, as shown by red dots in

15 figure $3 \mathrm{a}$. Moreover, the interface exhibits a clear optical contrast without the appearance of diffuse layer, as shown in figure $4 \mathrm{f}$. These observations confirm the presence of charged dextran sulfate is crucial for jet widening.

Besides the unfolding and widening of the all-aqueous jets, 20 surprisingly, the diameter of the charged viscous jet becomes highly sensitive to changes in the flow rate of the continuous phase $Q_{\text {out }}$. While the diameter of the uncharged jet varies little in response to changes in $Q_{\text {out }}$ (see square symbols in figure 5), the diameter of the charged jet is significantly reduced when $Q_{\text {out }}$ is

25 increased, as shown by the triangular symbols in figure 5 . At a given applied voltage and thus a constant electrostatic repulsion among the charged dextran sulfate near the surface of the jet, an increase in the flow rate of the continuous phase leads to a larger viscous force that causes thinning of the jet. The higher 30 sensitivity to changes in $Q_{\text {out }}$ allows us to conveniently adjust the jet diameter by varying the flow rate of the continuous phase $Q_{\text {out }}$ at relatively higher voltages.

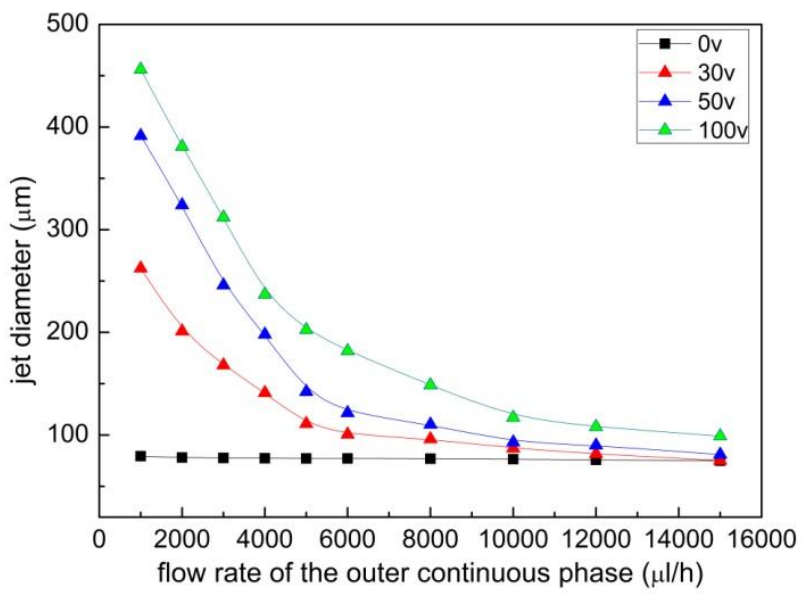

Fig.5 A plot of the jet diameter as a function of the flow rate of 35 the continuous phase $Q_{\text {out }}$. When the applied voltage increases, the jet diameter is more sensitive to changes in the flow rate of the continuous phase $Q_{\text {out }}$. In the plot, $Q_{i n}=250 \mu \mathrm{L} / \mathrm{h}$.

\section{Conclusions}

In conclusion, we present an electrical charging approach for 40 controlled deformation of viscous all-aqueous jets in microfluidic devices. A highly viscous jet of the dextran/dextran sulfate solution maintains a nearly constant diameter despite changes in the flow rate of the continuous phase. The insensitivity of the jet diameter to changes in flow rates makes it rather difficult, if not 45 impossible, to precisely modulate the jet diameter by hydrodynamic force alone. Upon applying a DC electric field, charged macromolecules migrate to the jet interface due to electrostatic repulsion. This electrical charging effect causes the jet to swell, and the resultant jet diameter is a result of the 50 delicate balance among electrostatic, viscous and interfacial tension forces. Our approach represents a first demonstration of electrostatic-induced deformation of viscous jets in an allaqueous platform ${ }^{[11,15]}$, with great potentials for generating biocompatible hydrogel fibers with tunable structures ${ }^{[16]}$ for 55 applications in cell culture and tissue engineering.

\section{Notes and references}

${ }^{a}$ Department of Mechanical Engineering, The University of Hong Kong, Pokfulam Road, Hong Kong.

${ }^{b} H K U$-Shenzhen Institute of Research and Innovation (HKU-SIRI), 60 Shenzhen, Guangdong, China

Tel: (852) 2859 7904; E-mail: ashum@hku.hk

$\dagger$ Electronic Supplementary Information (ESI) available: [details of state diagram in figure $\mathrm{S} 1$; the jet of the dextran solution changes diameters upon tuning the $\mathrm{Q}_{\text {out }}$ in figure $\mathrm{S} 2$; the critical voltage $\mathrm{Vc}^{*}$ at different $\mathrm{Q}_{\text {in }}$ 65 in figure S3]. See DOI: 10.1039/b000000x/

$\$$ This research was supported by the Early Career Scheme (HKU 707712P) from the Research Grants Council of Hong Kong, the Basic Research Program-General Program (JC201105190878A) from the Science and Technology Innovation Commission of Shenzhen 70 Municipality, as well as the Seed Funding Programme for Basic Research (201101159009) and Small Project Funding (201109176165) from the University of Hong Kong.

1 a) P. D. Dalton, L. Flynn and M. S. Shoichet, Biomaterials, 2002, 23 , 75 3843-3851; b) M. Hu, M. Kurisawa, R. Deng, C.-M. Teo, A. Schumacher, Y.-X. Thong, L. Wang, K. M. Schumacher and J. Y. Ying, Biomaterials, 2009, 30, 3523-3531.

2 a) S. Yang, K. F. Leong, Z. Du and C. K. Chua, Tissue engineering, 2001, 7, 679-689; b) L. Flynn, P. D. Dalton and M. S. Shoichet, Biomaterials, 2003, 24, 4265-4272.

3 D. Li and Y. Xia, Advanced materials, 2004, 16, 1151-1170.

4 N. E. Fedorovich, J. R. De Wijn, A. J. Verbout, J. Alblas and W. J. A. Dhert, Tissue Engineering Part A, 2008, 14, 127-133.

5 J. Yeh, Y. Ling, J. M. Karp, J. Gantz, A. Chandawarkar, G. Eng, J.

85 Blumling Iii, R. Langer and A. Khademhosseini, Biomaterials, 2006, 27, 5391-5398.

6 H. Geckil, F. Xu, X. Zhang, S. Moon and U. Demirci, Nanomedicine, 2010, 5, 469-484.

7 a) A. Gunther and K. F. Jensen, Lab on a Chip, 2006, 6, 1487-1503;

90 b) P. J. A. Kenis, R. F. Ismagilov and G. M. Whitesides, Science, 1999, 285, 83-85.

8 D. M. Brunette and J. E. Till, Journal of Membrane Biology, 1971, 5, 215-224.

9 a) D. Forciniti, C. K. Hall and M. R. Kula, Journal of Biotechnology, 1990, 16, 279-296; b)I. Z. Sam D. Geschiere, Volkert van Steijn, Ger J. M. Koper, Jan H. van Esch, and Michiel T. Kreutzer, Biomicrofluidics, 2012, 6, 022007.

10 H. C. Shum, J. Varnell and D. A. Weitz, Biomicrofluidics, 2012, 6, 012808-012809.

10011 Z. Nie, M. Seo, S. Xu, P. Lewis, M. Mok, E. Kumacheva, G. Whitesides, P. Garstecki and H. Stone, Microfluid Nanofluid, 2008, 5, 585-594.

12 a) T. Cubaud, B. M. Jose and S. Darvishi, Physics of Fluids, 2011, 23, 042002-042009. b) T. Cubaud and T. G. Mason, Soft Matter 2012, 8, 10573-10582.

13 R. Lindken, M. Rossi, Gro and J. Westerweel, Lab on a Chip, 2009, 9, 2551-2567.

14 a) G. Münchow, S. Hardt, J. P. Kutter and K. Drese, Lab on a Chip, 2007, 7, 98-102; b) G. Münchow, S. Hardt, J. P. Kutter and K.

110 Drese, Journal of the Association for Laboratory Automation, 2006 11, 368-373; c) Y. Song and H. C. Shum, Langmuir, 2012, 28, 12054-12059;

15 a) C.-H. Choi, H. Yi, S. Hwang, D. A. Weitz and C.-S. Lee, Lab on a Chip, 2011, 11, 1477-1483; b) V. Breedveld, A. P. Nowak, J. Sato, 115 T. J. Deming and J. David, Macromolecules, 2004, 37, 3943-3953. 